\title{
circSAMD4A participates in the apoptosis and autophagy of dopaminergic neurons via the miR-29c-3p-mediated AMPK/mTOR pathway in Parkinson's disease
}

\author{
WENSHENG WANG, RONGXIANG LV, JINGJING ZHANG and YU LIU \\ Department of Neurology, Ningbo No. 6 Hospital, Ningbo, Zhejiang 315040, P.R. China
}

Received August 27, 2020; Accepted April 9, 2021

DOI: $10.3892 / \mathrm{mmr} .2021 .12179$

\begin{abstract}
Parkinson's disease (PD) can lead to movement injury and cognitive dysfunction. Although advances have been made in attenuating PD, the effect of inhibiting the development of PD remains disappointing. Therefore, the present study aimed at investigating the etiology of Parkinson's disease and developing an alternative therapeutic strategy for patients with PD. A PD mouse model was established using an intraperitoneal injection of 1-methyl-4-phenyl-1,2,3,6-tetrahydropyridine hydrochloride (MPTP-HCl; $30 \mathrm{mg} / \mathrm{kg}$ /day for 5 days), and a PD cellular model was established by treating SH-SY5Y cells with different concentrations of 1-methyl-4-phenylpyridinium $\left(\mathrm{MPP}^{+}\right)$for $24 \mathrm{~h}$. The expression levels of circular RNA sterile $\alpha$ motif domain containing 4A (circSAMD4A) and microRNA (miR)-29c-3p in both midbrain tissues and SH-SY5Y cells were detected via reverse transcription-quantitative PCR. The interaction between circSAMD4A and miR-29c-3p was verified using a dual-luciferase reporter experiment. Apoptosis-, autophagy- and 5'AMP-activated protein kinase (AMPK)/mTOR cascade-associated proteins in midbrain tissues and SH-SY5Y cells were detected using western blotting. Furthermore, TUNEL staining and flow cytometry were used to analyze cell apoptosis. It was found that circSAMD4A was upregulated, while miR-29c-3p was downregulated in both PD animal and cellular models. Moreover, circSAMD4A directly targeted and negatively regulated miR-29c-3p. Further studies identified that circSAMD4A knockdown inhibited MPTPor $\mathrm{MPP}^{+}$-induced apoptosis and autophagy; however, these
\end{abstract}

Correspondence to: Dr Wensheng Wang, Department of Neurology, Ningbo No. 6 Hospital, 1059 Zhongshan East Road, Yinzhou, Ningbo, Zhejiang 315040, P.R. China

E-mail: wangwensh10@163.com

Abbreviations: circRNAs/circs, circular RNAs; PD, Parkinson's disease; MPTP-HCl, 1-methyl-4-phenyl-1,2,3,6-tetrahydropyridine hydrochloride; RT-qPCR, reverse transcription-quantitative PCR; AMPK, 5'AMP-activated protein kinase; miR, microRNA

Key words: AMPK/mTOR, apoptosis, autophagy, circ-sterile $\alpha$ motif domain containing 4A, miR-29c-3p, PD effects were abolished by an miR-29c-3p inhibitor. In addition, circSAMD4A knockdown repressed phosphorylated-AMPK expression and increased mTOR expression in MPTP- or $\mathrm{MPP}^{+}$-induced PD models, the effects of which were reversed by a miR-29c-3p inhibitor. Collectively, these results suggested that circSAMD4A participated in the apoptosis and autophagy of dopaminergic neurons by modulating the AMPK/mTOR cascade via miR-29c-3p in PD.

\section{Introduction}

Parkinson's disease (PD) is one of the most frequent degenerative disorders of the nervous system, and is characterized by the large-scale loss of dopaminergic neurons of the substantia nigra pars compacta, as well as the gradual accumulation of intracellular $\alpha$-synuclein (1). In general, PD occurs frequently amongst the elderly; however, previous studies have shown that PD is occurring earlier compared with during past decades (2-4). It has been determined that $\sim 18$ new PD cases will occur per year in a population of 1,010,000 (5). Abnormal nerve signals caused by PD lead to a range of movement impairments and cognitive deficits, thereby greatly reducing the quality of life of patients with PD and significantly increasing the medical and economic burden on society (6). Although current treatments have made progress in relieving PD symptoms, the effects on inhibiting PD development remain disappointing (7). It is therefore essential to conduct studies aimed at understanding the etiology of PD and developing more effective therapeutic options for patients with PD.

Circular RNAs (circRNAs/circs) comprise one of the most common subtypes of non-coding RNAs, which are ubiquitously present in mammal cells and characterized by loop structures (8). Unlike classic linear RNA molecules, circRNAs have no 5'-cap and 3'-poly A tail, which makes circRNAs resistant to endonucleases (9). circRNAs can therefore exist in a cellular environment for a relatively long time and may be used as promising diagnostic markers for multiple human diseases including breast cancer, colorectal cancer and hepatocellular carcinoma $(10,11)$. circRNAs play a key role in numerous cellular processes, including apoptosis, autophagy, proliferation and differentiation (10). Moreover, aberrant circRNA expression profiles have been associated with the initiation and progression of various human diseases, especially cancer (12). In recent years, circRNAs were 
reported to be responsible for the pathogenesis of multiple acute neurodegenerative disorders, including cerebral ischemia, stroke and epilepsy (13-15). Its role in Alzheimer's disease (AD) has also been demonstrated by accumulating evidence $(16,17)$. However, the role of circRNAs has rarely been studied in PD.

circ-sterile $\alpha$ motif domain containing 4A (circSAMD4A; ID, hsa_circ_0004846) was originally reported by Zhao and Jing (18) to facilitate osteosarcoma cell proliferation by regulating the microRNA (miRNA/miR)-1244/MDM2 proto-oncogene axis. The results from our preliminary experiments revealed that circSAMD4A was markedly increased in 1-methyl-4-phenylpyridinium $\left(\mathrm{MPP}^{+}\right)$-induced SH-SY5Y cells, indicating that circSAMD4A may serve a role in the pathogenesis of PD (data not shown). The present study therefore aimed to characterize the role and mechanism of circSAMD4A in PD, to further identify the pathogenesis of $\mathrm{PD}$ and to develop more effective therapeutic targets for patients with PD.

\section{Materials and methods}

PD animal model. All animal experiments were conducted following stated protocols from the Research Committee of Ningbo No. 6 Hospital and permission was obtained from the Animal Experiment Center of the Institute of Radiation Medicine of the Chinese Academy of Medical Sciences [permit no. SCXK(JING)2019-0010].

A total of 36 male C57BL/6J mice (10 weeks, $22 \pm 2 \mathrm{~g}$ ) were obtained from the Animal Experiment Center of the Institute of Radiation Medicine of the Chinese Academy of Medical Sciences. Animals were kept in separate cages at $23 \pm 2^{\circ} \mathrm{C}$ under controlled humidity (45-65\%) with free access to food and water under a 12-h light/dark cycle, and were randomly divided into PD $(n=30)$ and sham groups $(n=6)$. A PD animal model was established using an intraperitoneal injection of 1-methyl-4-phenyl-1,2,3,6-tetrahydropyridine hydrochloride (MPTP-HCl; 30 mg/kg/day; Sigma-Aldrich; Merck KGaA) for 5 consecutive days as previously described (19). Sham animals were given an equivalent volume of sterile saline. Mice were decapitated at $0,1,3,5$ and 7 days after the last injection, and the midbrain tissue was isolated and stored at $-80^{\circ} \mathrm{C}$. The experiment was completed between January 2019 and December 2019.

For the exogenous delivery of circSAMD4A in PD animals, all mice were divided into six groups $(n=6)$ at random: Negative control (NC) group (saline); $\mathrm{MPTP}-\mathrm{HCl}$ group (MPTP group); MPTP + NC short hairpin (sh)RNAs (shNC) group; MPTP + circSAMD4A shRNAs (sh-circ) group; MPTP + sh-circ + NC miR-29c-3p inhibitor (NC inhibitor) group; and the MPTP + sh-circ + miR-29c-3p inhibitor group. The sh-circ and shNC were purchased from Promega Corporation. The mice were anesthetized using 2\% isoflurane (20), then fixed in a stereotaxic frame (Stoelting, Co.), which was followed by disinfecting the skin near the brain with alcohol, cutting open the skull surface, ascertaining the stereotaxic coordinates of dorsal hippocampus $(\mathrm{AP}=-5.4 \mathrm{~mm}$; $\mathrm{ML}=+1.7 \mathrm{~mm}$; DV=-7.5 mm) and drilling a hole. Then sh-circ, miR-29c-3p inhibitor or a matched control was intraperitoneally injected into the hippocampus $(0.3 \mu \mathrm{l}$ per site) via a tip needle
(33 G) with a syringe (10 $\mu$; Hamilton Company). At 3 days after vector injection, the injections of MPTP-HCl or sterile saline were conducted in the same manner, as aforementioned.

During the course of the experiment, the mice were monitored daily for their health and specific behaviors, including reduced locomotor activity and abnormal postures and squeals. Losing $20 \%$ of body weight was considered the humane end point for euthanasia. At the end of the experiment, mice were put into the euthanasia chamber, which was then filled with $\mathrm{CO}_{2}$ with a displacement rate of $20 \%$ chamber volume/min. When the mice were unconscious and stopped breathing, the $\mathrm{CO}_{2}$ flow was maintained for $1 \mathrm{~min}$. The death of the mice was confirmed by cardiac arrest and no reaction to the toe pinch reflex (21). Then, cervical dislocation was adopted to determine death.

Cell lines and establishment of a PD cell model. SH-SY5Y and 293 cell lines were obtained from the American Type Culture Collection, and were cultured in DMEM (Gibco; Thermo Fisher Scientific, Inc.), supplemented with $10 \%$ FBS and $100 \mathrm{U} / \mathrm{ml}$ penicillin/streptomycin in a humidified atmosphere with $5 \% \mathrm{CO}_{2}$ at $37^{\circ} \mathrm{C}$. To establish the PD cell model, SH-SY5Y cells were incubated with $\mathrm{MPP}^{+}(0.25,0.5$ or $1 \mathrm{mM}$ ) for $24 \mathrm{~h}$ at $37^{\circ} \mathrm{C}$.

Cell transfection and luciferase reporter (DLR) assay. miR-29c-3p mimics (including miR control, cat. no. HMI0439, concentration: $\geq 10^{6} \mathrm{TU} / \mathrm{ml}$ ), miR-29c-3p inhibitor (including miR-29c-3p inhibitor NC, cat. no. HSTUD0440, concentration: $\geq 10^{6} \mathrm{TU} / \mathrm{ml}$ ) were purchased from Sigma-Aldrich (Shanghai) Trading Co., Ltd., shNC (cat. no. C01001, concentration: $10^{8} \mathrm{TU} / \mathrm{ml}$ ), sh-circ (cat. no. C03002, concentration: $10^{8} \mathrm{TU} / \mathrm{ml}$ ), pcDNA empty vector (cat. no. G01001, concentration: $10^{8} \mathrm{TU} / \mathrm{ml}$ ), and pcDNA-circSAMD4A (cat. no. G04002, concentration: $10^{8} \mathrm{TU} / \mathrm{ml}$ ) were purchased from Shanghai GenePharma Co., Ltd. The sequences of miR-29c-3p mimics were: (5'-3') sense UAGCACCAUUUGAAAUCGG UUA, and antisense ACCGAUUUCAAAUGGUGCUAUU; mir-29c-3p inhibitor: (5'-3') UAACCGAUUUCAAAUGGU GCUA; sh-circ: (5'-3') AGCACAAGTACAAGAATCATT; miRNA mimic NC: (5'-3') sense UUUGUACUACACAAA AGUACUG and (5'-3') antisense CAGUACUUUUGUGUA GUACAAA; miRNA inhibitor NC: (5'-3') CAGUACUUUUGU GUAGUACAAA. The cell transfection was performed using Lipofectamine ${ }^{\circledR} 3000$ (Invitrogen; Thermo Fisher Scientific, Inc.) according to manufacturer's instructions for $48 \mathrm{~h}$ at $37^{\circ} \mathrm{C}$. Then, cells were treated with $1 \mathrm{mM} \mathrm{MPP}^{+}$solution for $24 \mathrm{~h}$ at $37^{\circ} \mathrm{C}$.

The binding site seed regions of miR-29c-3p and 3'-UTR of circSAMD4 were determined using TargetScan (release 7.2, http://www.targetscan.org). A pmirGLO vector (Guangzhou RiboBio Co., Ltd.) containing wild-type (WT) and mutant (MUT) 3'-UTR of circSAMD4A was constructed according to the predicted binding sites. Then, cells were co-transfected with the circSAMD4A 3'-UTR-WT reporter vector or 3'-UTR-MUT reporter vector using Lipofectamine ${ }^{\circledR} 3000$ (Invitrogen; Thermo Fisher Scientific, Inc.) for $48 \mathrm{~h}$. Subsequently, a dual-reporter luciferase assay system in a Tecan SpectraFluorPlus plate-reader (Tecan Group, Ltd.) was employed to determine luciferase activity. 
Table I. Primer sequences used in reverse transcription-quantitative PCR assay.

\begin{tabular}{ll}
\hline Gene ID & \multicolumn{1}{c}{ Sequence $\left(5^{\prime}-3^{\prime}\right)$} \\
\hline hsa-GAPDH & Forward: TGTTCGTCATGGGTGTGAAC \\
mmu-GAPDH & Reverse: ATGGCATGGACTGTGGTCAT \\
mmu_circSAMD4A & Forward: AGGTCGGTGTGAACGGATTTG \\
& Reverse: GGGGTCGTTGATGGCAACA \\
hsa_circSAM4A & Forward: TATGTTGTGGATCCTGTTCGGCAAC \\
mmu/hsa-miR-29c-3p & Reverse: TGGTGGTAGACCAAGACTTGTGAT \\
& Forward: GCTCCTGATGGTCACCTTGT \\
& Reverse: TCACCACTCCTGGTTCTTCC \\
mmu-U6 & RT: GTTGGCTCTGGTGCAGGGTCCGAGGTATTCGCAC \\
& CAGAGCCAACTAACCG \\
& Forward: TAGCACCATTTGAAATCGGTTA \\
& Reverse: GTGCAGGGTCCGAGGTATTC \\
& RT: GTTGGCTCTGGTGCAGGGTCCGAGGTATTCGCAC \\
hsa-U6 & CAGAGCCAACAAAAATATGG \\
& Forward: CGCAAGGATGACACGCAAAT \\
& Reverse: GTGCAGGGTCCGAGGTATTC \\
& RT: GTTGGCTCTGGTGCAGGGTCCGAGGTATTCGCACCAG \\
& AGCCAACAAAATATGG \\
& Forward: GCTTCGGCAGCACATATACT \\
& Reverse: GTGCAGGGTCCGAGGTATTC \\
&
\end{tabular}

miR, microRNA; circSAMD4A, circular RNA sterile $\alpha$ motif domain containing 4A.

$R N A$ extraction and reverse transcription-quantitative $P C R$ $(R T-q P C R)$. Total RNAs of midbrain tissues and SH-SY5Y cells were isolated using TRIzol ${ }^{\circledR}$ (Invitrogen; Thermo Fisher Scientific, Inc.), followed by the evaluation of its quality and concentration using a SMA $400 \mathrm{UV}$-vis spectrophotometer (Merinton Instrument, Ltd.). RNA was subsequently transcribed into cDNA using a RevertAid First Strand cDNA Synthesis kit (cat. no. K1622, Invitrogen; Thermo Fisher Scientific, Inc.) according to the manufacturer's instructions. A SYBR Green PCR kit was used for RT-qPCR on a CFX96 real-time PCR system (Bio-Rad Laboratories, Inc.) with the thermocycling conditions: Initial denaturation at $95^{\circ} \mathrm{C}$ for $10 \mathrm{~min}, 40$ cycles of denaturation at $95^{\circ} \mathrm{C}$ for $15 \mathrm{sec}$, annealing at $61^{\circ} \mathrm{C}$ for $40 \mathrm{sec}$ and elongation at $72^{\circ} \mathrm{C}$ for $40 \mathrm{sec}$. Final extension step was at $72^{\circ} \mathrm{C}$ for $5 \mathrm{~min}$. U6 was used as the internal control of miR-29c-3p, and GAPDH was applied as the internal control of mRNAs. U6 was used as the internal control of miR-29c-3p, and GAPDH was applied as the internal control of mRNAs. The expression levels of GAPDH or U6 were used to calculate the $\mathrm{Cq}$ value based on the $2^{-\Delta \Delta \mathrm{Cq}}$ method (22). The primers sequences used in this study appear in Table 1.

Western blot analysis. Total proteins of midbrain tissues and SH-SY5Y cells were isolated using RIPA buffer (Beyotime Institute of Biotechnology), and the concentration was estimated using a Bradford Protein Assay kit (Beyotime Institute of Biotechnology). Proteins (30 $\mu \mathrm{g} /$ lane) were loaded into and separated by $10 \%$ SDS-PAGE, and then transferred onto PVDF membranes (EMD Millipore). After blocking in $5 \%$ non-fat milk for $2 \mathrm{~h}$ at room temperature, the membrane was incubated with primary antibodies at $4{ }^{\circ} \mathrm{C}$ overnight. The membrane was then incubated with a corresponding HRP-labeled donkey anti-rabbit secondary antibody (1:3,000; cat. no. ab6802; Abcam) for $1 \mathrm{~h}$ at room temperature. Finally, the blots were visualized with an ECL kit (cat. no. ab133406; Abcam). GAPH was used as an internal control. The primary antibodies included: rabbit anti-caspase-3 (1:1,000, cat. no. ab49822; Abcam), rabbit anti-Bcl2 (1:2,000; cat. no. ab196495; Abcam), rabbit anti-Bax (1:3,000, cat. no. 2772; Cell Signaling Technology, Inc.), rabbit anti-GAPDH (1:5,000; cat. no. ab125247; Abcam), LC3II (rabbit; 1:3,000; cat. no. ab229327; Abcam), Beclin (rabbit; 1:5,000; cat. no. ab62557; Abcam), phosphorylated (p)-5'AMP-activated protein kinase (AMPK; rabbit; 1:1,000; cat. no. ab23875; Abcam), AMPK (rabbit; 1;5,000; cat. no. ab3760; Abcam), p-mTOR (rabbit; 1:10,000; cat. no. ab137133; Abcam) and mTOR (rabbit; 1:10,000; cat. no. ab109268; Abcam). Subsequently, the membranes were incubated with an anti-rabbit IgG secondary antibody (1:1,000, cat. no. sc-2357; Santa Cruz Biotechnology, Inc.) for $2 \mathrm{~h}$ at room temperature. Protein were visualized using ChemiDoc XRS System (Bio-Rad Laboratories, Inc.) equipped with a 12-bit digital camera coupled to the Quantity One software (version 4.6.3; Bio-Rad Laboratories, Inc.).

TUNEL assay. The midbrain tissue and treated SH-SY5Y cells were fixed using $4 \%$ paraformaldehyde for $30 \mathrm{~min}$ at $25^{\circ} \mathrm{C}$, then dehydrated, paraffin-embedded and sectioned $(5 \mu \mathrm{m})$. Slides were covered with VECTASHIELD ${ }^{\circledR}$ mounting medium (Vector Laboratories, Inc.) with DAPI. Subsequently, the slices were incubated with proteinase $\mathrm{K}(10 \mu \mathrm{g} / \mathrm{ml}$; 
A

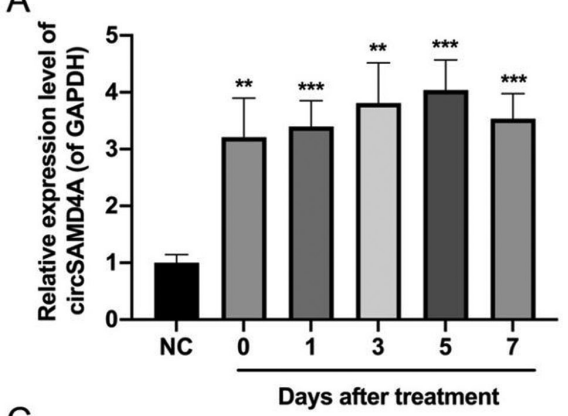

C

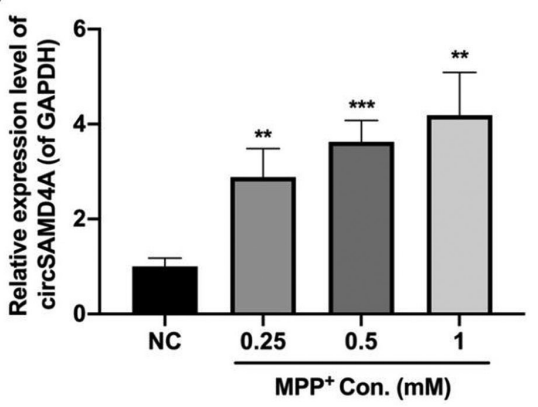

B

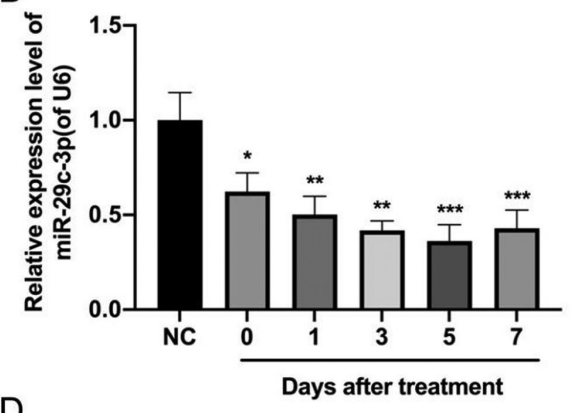

D

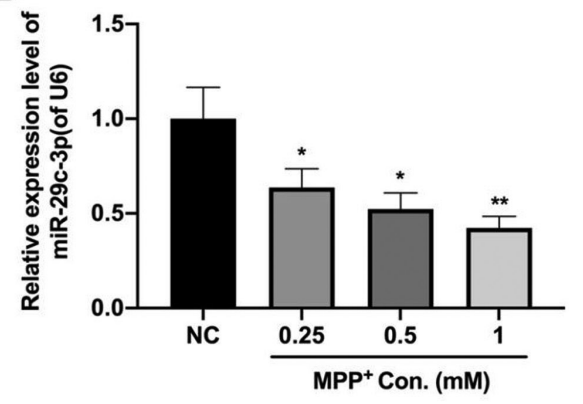

Figure 1. circSAMD4A and miR-29c-3p are dysregulated in PD. The expression levels of circSAMD4A and miR-29c-3p were determined using reverse transcription-quantitative PCR in (A and B) a MPTP-induced PD animal model and (C and D) $\mathrm{MPP}{ }^{+}$-treated SH-SY5Y cells. ${ }^{*} \mathrm{P}<0.05,{ }^{* *} \mathrm{P}<0.01,{ }^{* * *} \mathrm{P}<0.001$ vs. NC. PD, Parkinson's' disease; Con, concentration; NC, negative control; miR, microRNA; circSAMD4A, circular RNA sterile $\alpha$ motif domain containing 4A; $\mathrm{MPP}^{+}, 1-$ methyl-4-phenylpyridinium.

Beyotime Institute of Biotechnology) for $10 \mathrm{~min}$ at $37^{\circ} \mathrm{C}$. The detection of apoptosis-specific nuclear DNA fragmentation was conducted using a Colorimetric TUNEL Apoptosis Assay kit (Beyotime Institute of Biotechnology) for $1 \mathrm{~h}$ at $37{ }^{\circ} \mathrm{C}$ in the dark. The TUNEL-positive cell nucleus was detected using an Axiovert 200 fluorescence microscope (magnification, x200; Olympus Corporation), and TUNEL-positive cells were observed under fluorescent microcopy (Olympus Corporation) and counted in six random fields.

Flow cytometry analysis. After being transfected with sh-circ, shNC, miR-29c-3p NC or miR-29c-3p inhibitor, SH-SY5Y cells were collected and washed three times with PBS. Cells were then resuspended in annexin $\mathrm{V}$ binding buffer, and cell apoptosis was analyzed using an annexin V-FITC Apoptosis Detection kit (Beyotime Institute of Biotechnology). The cells were incubated with $5 \mu \mathrm{l}$ Annexin V-FITC and $10 \mu \mathrm{l}$ PI for $20 \mathrm{~min}$ at $25^{\circ} \mathrm{C}$ in the dark. Cell apoptosis was analyzed using a flow cytometer FACSCanto II (version 6.13; BD Biosciences). The apoptotic rate (\%) was calculated as the percentage of the early + late apoptotic cells.

Luciferase reporter assay. The fragment of circSAMD4A containing the wild-type (WT) or mutant (MUT) of the miR-29c-3p binding site was amplified and inserted into a PGL3 luciferase vector (Promega Corporation), to construct the luciferase reporter vectors, PGL3-circSAMD4A-WT and PGL3-circSAMD4A-MUT. 293 cells were co-transfected with $100 \mathrm{ng}$ constructed luciferase reporter vector and $50 \mathrm{nM}$ miR-29c-3p mimic using Lipofectamine 3000 at $37^{\circ} \mathrm{C}$ for $48 \mathrm{~h}$. After $48 \mathrm{~h}$ of incubation, 293 cells were harvested to determine the luciferase activity using a Dual-Glo Luciferase assay kit (Promega Corporation). Relative luciferase activity of the 3'UTR reporter constructs was normalized by Renilla activity.

Statistical analysis. All the experiments were performed independently three times, and data are presented as the mean \pm SD. The data were analyzed using GraphPad Prism, version 7.0 software (GraphPad Software, Inc.). Comparisons between two groups were analyzed using paired Student's t-test. Comparisons between different groups were analyzed using one-way ANOVA with Tukey's test. $\mathrm{P}<0.05$ was considered to indicate a statistically significant difference.

\section{Results}

circSAMD4A and miR-29c-3p are dysregulated in PD. To investigate whether circSAMD4A and miR-29c-3p served a role during the pathogenesis of $\mathrm{PD}$, their expression levels were measured in both PD animals and cellular models using RT-qPCR. The results indicated that the expression level of circSAMD4A was upregulated, while miR-29c-3p was downregulated in the PD animal model (Fig. 1A and B). Consistently, $\mathrm{MPP}^{+}$treatment increased circSAMD4A expression but decreased miR-29c-3p expression in SH-SY5Y cell lines (Fig. 1C and D). Taken together, these results suggested that circSAMD4A and miR-29c-3p served a role in the pathogenesis of PD.

circSAMD4A targets and negatively regulates $m i R-29 c-3 p$. To further study the relationship of circSAMD4A and miR-29c-3p in the pathogenesis of PD, it was predicted whether there was a complementary sequence between circSAMD4A and miR-29c-3p using TargetScan software (release 7.2, http://www.targetscan.org). Fig. 2A (upper panel) 
A

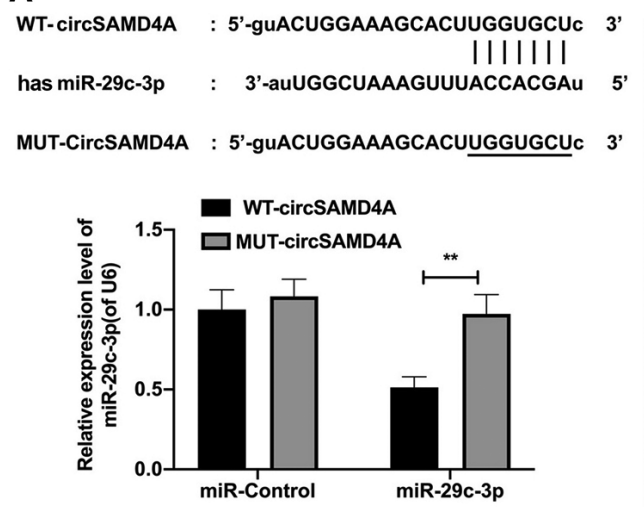

B

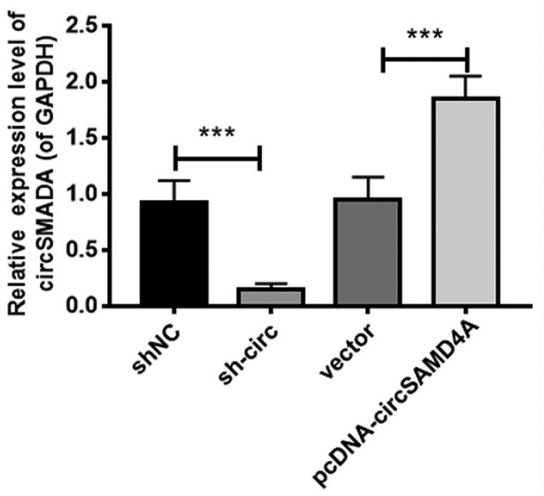

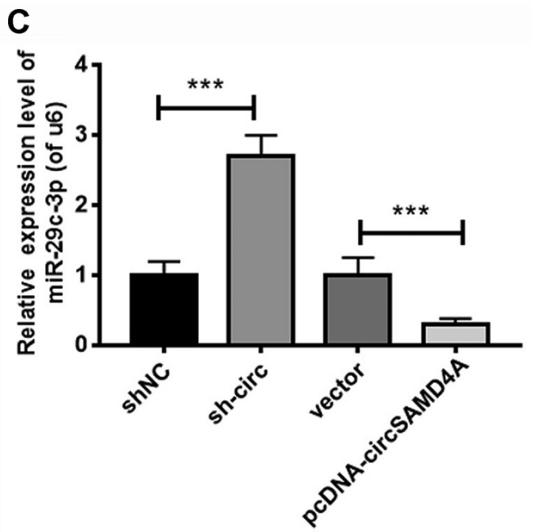

Figure 2. circSAMD4A targets and negatively regulates miR-29c-3p. (A) Upper panel, TargetScan analysis showing the putative recognition sequence of miR-29c-3p in circSAMD4A. Lower panel, a dual-luciferase reporter was used to verify the interaction between circSAMD4A and miR-29c-3p in 293 cells. The relative expression levels of (B) circSAMD4A and (C) miR-29c-3p were detected in SH-SY5Y cells transfected with sh-circ, pcDNA-circSAMD4A or matched controls via reverse transcription-quantitative PCR. ${ }^{* *} \mathrm{P}<0.01,{ }^{* * *} \mathrm{P}<0.001$. sh-circ, sh-circSAMD4A; miR, microRNA; circSAMD4A, circular RNA sterile $\alpha$ motif domain containing 4A; WT, wild-type; MUT, mutant; sh, short hairpin RNA; NC, negative control.

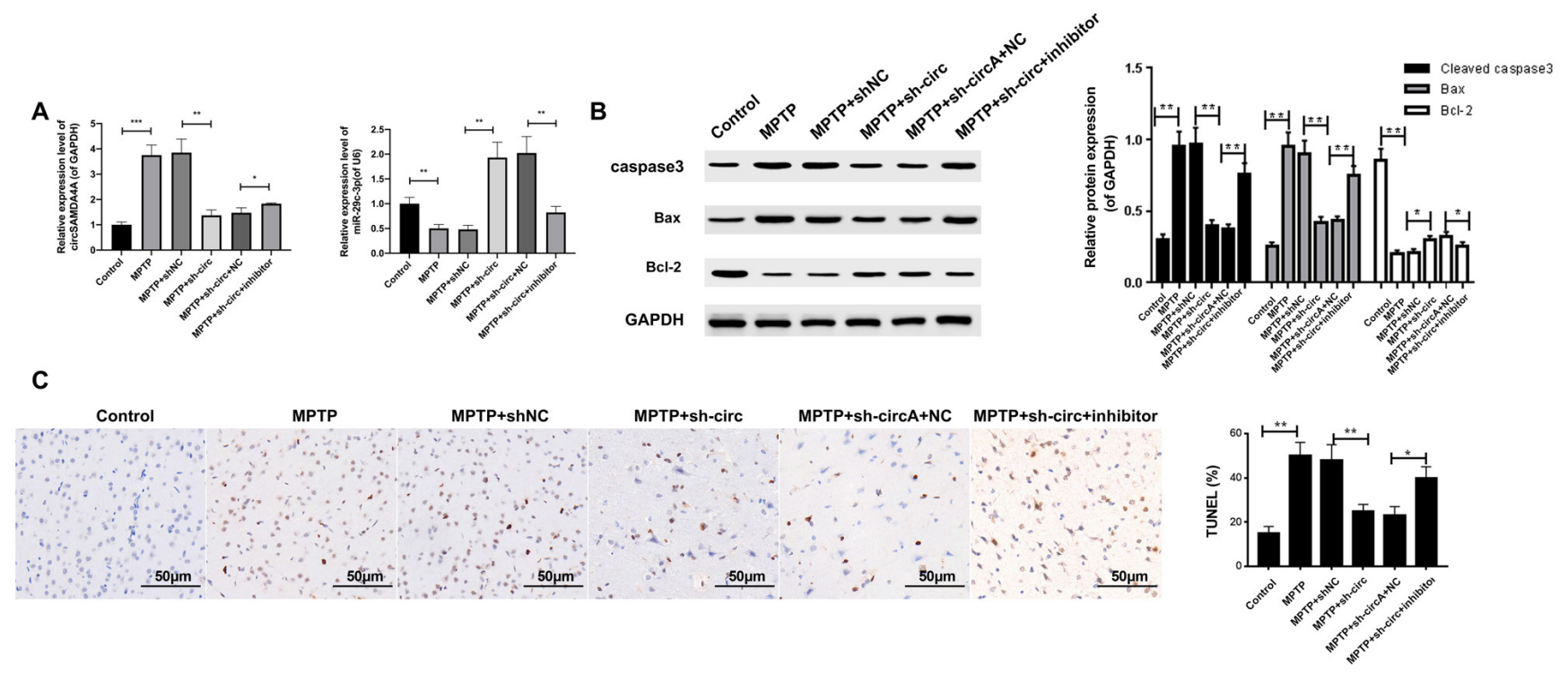

Figure 3. A miR-29c-3p inhibitor abrogates the protective effects of sh-circ against MPTP-induced cell apoptosis. (A) Expression levels of circSAMD4A and miR-29c-3p in sh-circ, shNC, miR-29c-3p NC or miR-29c-3p inhibitor-injected MPTP-induced mice were measured via reverse transcription-quantitative PCR. (B) Western blotting was used to determine protein expression levels of caspase 3, Bcl2 and Bax in transfected mice. GAPDH or U6 were used as an internal control. (C) Apoptotic cells in treated mice were analyzed using the TUNEL assay. ${ }^{*} \mathrm{P}<0.05,{ }^{* * *} \mathrm{P}<0.01,{ }^{* * * *} \mathrm{P}<0.001$. sh-circ, sh-circSAMD4A; miR, microRNA; circSAMD4A, circular RNA sterile $\alpha$ motif domain containing 4A; sh, short hairpin RNA; NC, negative control; MPTP, 1-methyl-4-phenyl-1,2,3,6-tetrahydropyridine.

shows the predicted recognition of the miR-29c-3p sequence in circSAMD4A transcripts. To verify the interaction between circSAMD4A and miR-29c-3p, the transfection efficiency of miR-29c-3p mimics in 293 cells was determined. The results demonstrated that miR-29c-3p expression was significantly increased in miR-29c-3p mimics group compared with that in miR-control group, suggesting the successful transfection of miR-29c-3p mimics in 293 cells (Fig. S1A). Next, a dual-luciferase reporter driven by WT-circSAMD4A or MUT-circSAMD4A was established and co-transfected into 293 cells with miR-29c-3p mimic or the miR-control. The results indicated that the luciferase activity of WT-circSAMD4A was decreased in miR-29c-3p-overexpressing 293 cells, while there was no effect of miR-29c-3p transfection on the luciferase activities of MUT-circSAMD4A (Fig. 2A; lower panel).
Next, circSAMD4A was knocked down and overexpressed in SH-SY5Y cells via transfection, and the results demonstrated that circSAMD4A was significantly downregulated in the sh-circ group compared with the shNC group; while it was upregulated in pcDNA-circSAMD4A group compared with the vector group, suggesting the successful transfection of circSAMD4A shRNAs and circSAMD4A-overexpressed plasmid (Fig. 2B). Moreover, miR-29c-3p expression was measured using RT-qPCR in sh-circ or pcDNA-circSAMD4A transfected cells, and it was found that the expression level of miR-29c-3p was significantly increased by sh-circ, while circSAMD4A overexpression inhibited miR-29c-3p expression in SH-SY5Y cells (Fig. 2C). Together, the results demonstrated that circSAMD4A directly targeted miR-29c-3p to inhibit its expression. 
A

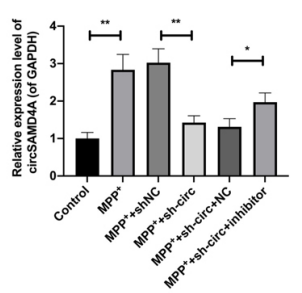

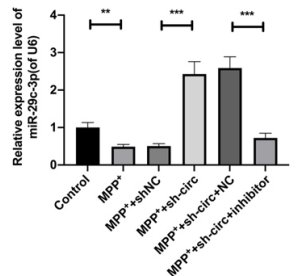

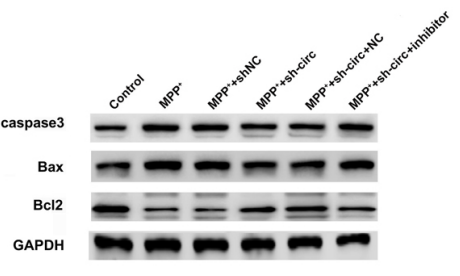

C

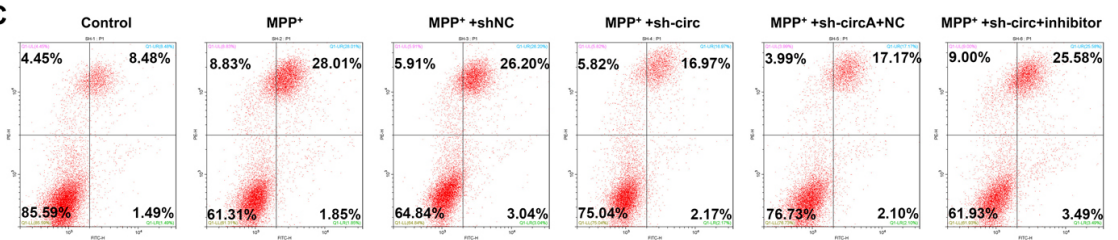

D

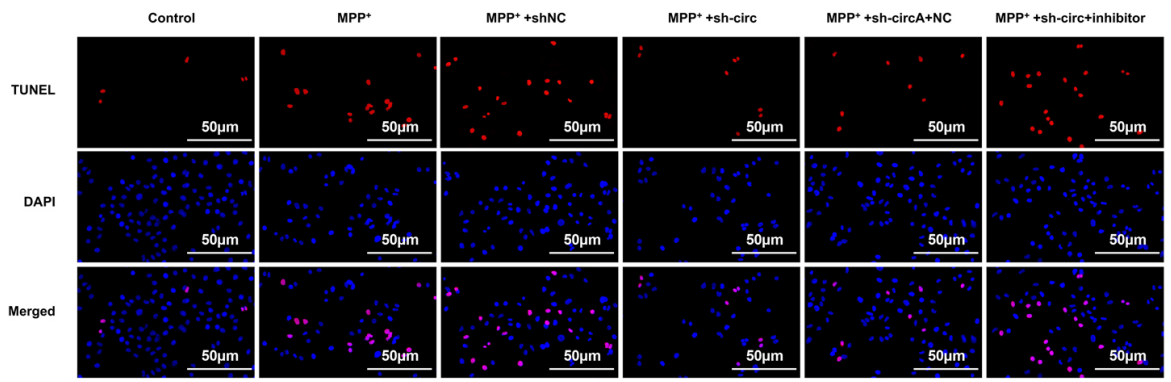

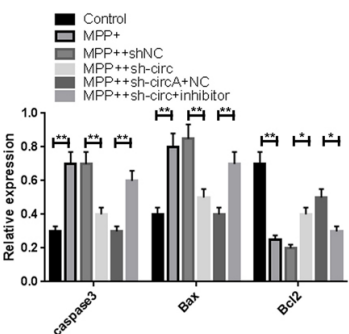
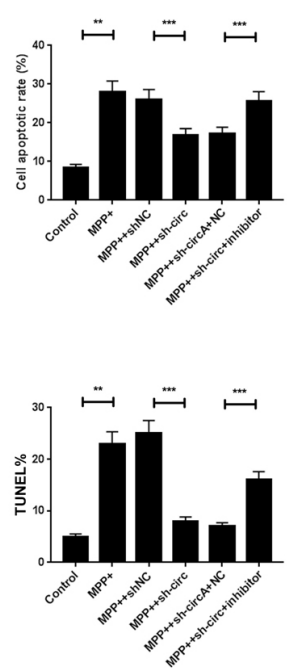

Figure 4. A miR-29c-3p inhibitor abrogates the protective effects of sh-circ against MPP+induced SH-SY5Y cell apoptosis. (A) Expression levels of circSAMD4A and miR-29c-3p were measured via reverse transcription-quantitative PCR SH-SY5Y cells treated with sh-circ, shNC, miR-29c-3p NC or miR-29c-3p inhibitor. (B) Western blotting was used to determine protein expression levels of caspase-3, Bcl2 and Bax in MPP+-induced SH-SY5Y cells treated with sh-circ, shNC, miR-29c-3p NC or miR-29c-3p inhibitor. GAPDH or U6 were used as an internal control. (C) Flow cytometry analysis and (D) TUNEL staining were performed to assess the effects of circSAMD4A knockdown and miR-29c-3p inhibitor on MPP+-induced apoptosis in SH-SY5Y cells. The apoptotic rate (\%) was calculated as the percentage of the early and late apoptotic cells. ${ }^{*} \mathrm{P}<0.05,{ }^{* *} \mathrm{P}<0.01,{ }^{* * *} \mathrm{P}<0.001$. sh-circ, sh-circSAMD4A; miR, microRNA; circSAMD4A, circular RNA sterile $\alpha$ motif domain containing 4A; sh, short hairpin RNA; NC, negative control; MPP ${ }^{+}$, 1-methyl-4-phenylpyridinium.

A miR-29c-3p inhibitor abrogates the protective effects of circSAMD4A knockdown against MPTP, and causes cell apoptosis in the PD animal model. After the interaction between circSAMD4A and miR-29c-3p was confirmed, the effect of circSAMD4A and miR-29c-3p on MPTP-induced apoptosis of dopaminergic neurons in $\mathrm{PD}$ mice was examined. The sh-circ, shNC, miR-29c-3p NC or miR-29c-3p inhibitor was intraperitoneally injected into the midbrain of mice. The RT-qPCR results indicated that sh-circ transfection resulted in downregulation of circSAMD4A but in the upregulation of miR-29c-3p (Fig. 3A). Moreover, it was found that co-transfection of sh-circ and miR-29c-3p inhibitor reversed the sh-circSAMD4A-induced upregulation of miR-29c-3p (Fig. 3A). In addition, the level of circSAMD4A was inhibited after co-transfection (Fig. 3A). The transfection effect of miR-29c-3p inhibitor was verified in PD mice (Fig. S1B).

Subsequently, the protein expression levels of caspase 3, $\mathrm{Bcl} 2$ and Bax were determined using western blotting. Compared with the control group, MPTP treatment significantly increased the expression levels of caspase- 3 and $\mathrm{Bcl} 2$, and decreased the expression level of Bax, while sh-circ transfection reversed the dysregulation of caspase-3, Bcl2 and Bax expression induced by MPTP (Fig. 3B), suggesting that sh-circ protected cells from MPTP-induced apoptosis. However, co-transfection of sh-circ and miR-29c-3p inhibitor abolished the protective effects of sh-circ against MPTP-induced cell apoptosis (Fig. 3B). In a similar manner, the TUNEL assay demonstrated that circSAMD4A knockdown significantly reduced MPTP-induced apoptosis of DA neurons, and the miR-29c-3p inhibitor abrogated the protective effect of circSAMD4A knockdown in the PD mice model (Fig. 3C). Collectively, these results indicated that the miR-29c-3p inhibitor abrogated the protective effect on cell apoptosis caused by circSAMD4A knockdown in the MPTP-induced PD animal model.

Knockdown of circSAMD4A exerts a protective effect on $M P P^{+}$-induced $S H-S Y 5 Y$ cell apoptosis by regulating $m i R-29 c-3 p$. After characterizing the role of circSAMD4A and miR-29c-3p in the PD animal model, the effects of the transfection of sh-circ or miR-29c-3p inhibitor on apoptosis were examined in the PD cell model. Moreover, the expression levels of circSAMD4A and miR-29c-3p in sh-circ- or miR-29c-3p-transfected SH-SY5Y cells in the presence of $\mathrm{MPP}^{+}$were determined. The results showed that sh-circ transfection abolished the upregulation of 
A

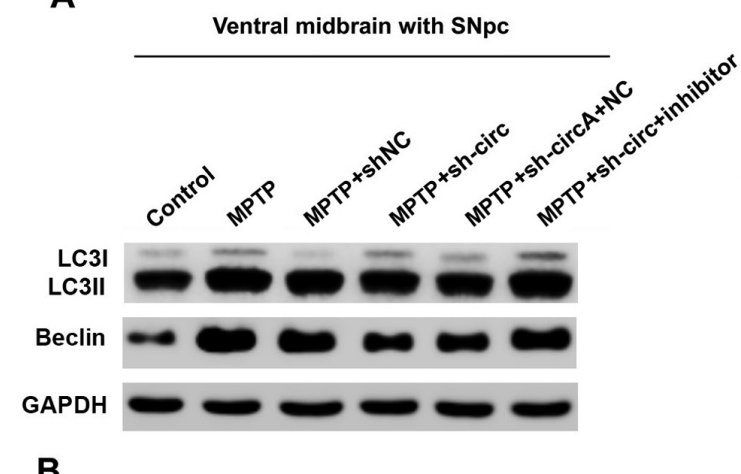

B

SH-SY5Y cells
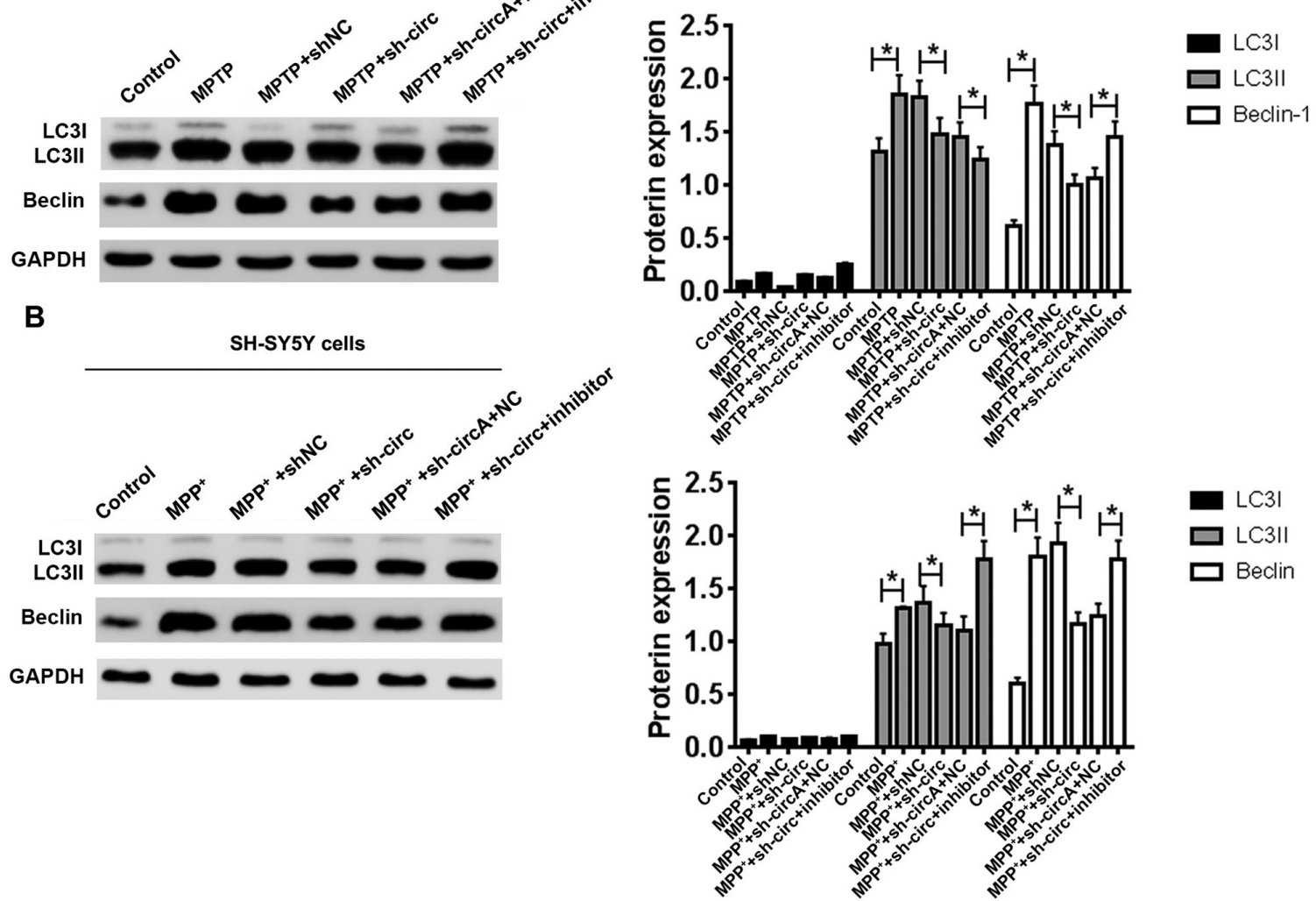

Figure 5. A miR-29c-3p inhibitor abrogates the repressive effects of sh-circ on autophagy. Western blotting was used to determine protein expressions of LC3II and Beclin in transfected (A) mice and (B) SH-SY5Y cells. GAPDH was used as an internal control. "P<0.05. sh-circ, sh-circSAMD4A; miR, microRNA; circSAMD4A, circular RNA sterile $\alpha$ motif domain containing 4A; sh, short hairpin RNA; NC, negative control; MPP+, 1-methyl-4-phenylpyridinium; MPTP, 1-methyl-4-phenyl-1,2,3,6-tetrahydropyridine; SNpc, substantia nigra pars compacta.

circSAMD4A and downregulation of miR-29c-3p induced by $\mathrm{MPP}^{+}$in $\mathrm{SH}-\mathrm{SY} 5 \mathrm{Y}$ cells. Moreover, this phenomenon was abrogated by the co-transfection of sh-circ and miR-29c-3p inhibitor (Fig. 4A).

The western blot analysis of apoptosis-related proteins demonstrated that sh-circ transfection reversed the upregulation of caspase- 3 and $\mathrm{Bcl} 2$ and downregulation of Bax induced by MPP ${ }^{+}$in SH-SY5Y cells; however, these effects of sh-circ were abolished by co-transfection of sh-circ and the miR-29c-3p inhibitor (Fig. 4B). Furthermore, flow cytometry analysis identified that circSAMD4A knockdown significantly repressed $\mathrm{MPP}^{+}$-induced SH-SY5Y cell apoptosis; however, co-transfection with the miR-29c-3p inhibitor abrogated this effect (Fig. 4C). Consistent results were obtained from TUNEL staining in SH-SY5Y cells transfected with sh-circ or the miR-29c-3p inhibitor (Fig. 4D). Thus, it was suggested that the miR-29c-3p inhibitor abrogated the protective effect of circSAMD4A knockdown against apoptosis in the $\mathrm{MPP}^{+}$-induced PD cell model.

The inhibitory effect of knockdown of circSAMD4A on autophagy is repressed by miR-29c-3p in both PD animals and cellular models. To determine whether the dysregulation of circSAMD4A and miR-29c-3p was associated with cell autophagy, the autophagy-related proteins were assessed in both the PD model animal and cells using western blotting. The results indicated that MPTP treatment activated in vivo autophagy as shown by the upregulation of LC3II and Beclin in the midbrain tissues of mice (Fig. 5A). It was found that knockdown of circSAMD4A inhibited the expression levels of LC3II and Beclin in the presence of MPTP compared with the shNC group; however, the miR-29c-3p inhibitor abolished this effect (Fig. 5A). Consistently, $\mathrm{MPP}^{+}$also activated in vitro autophagy as shown by the upregulation of LC3II and Beclin in SH-SY5Y cells (Fig. 5B). Knockdown of circSAMD4A significantly decreased the expression levels of LC3II and Beclin in the presence of $\mathrm{MPP}^{+}$in SH-SY5Y cells compared with the shNC group; however, the miR-29c-3p inhibitor abolished this effect (Fig. 5B). Taken together, these results indicated that the miR-29c-3p inhibitor abrogated the repressive effects of sh-circ on autophagy in both PD animal and cellular models.

A miR-29c-3p inhibitor abrogates the regulatory effects of circSAMD4A knockdown on the AMPK/mTOR pathway in vitro and in vivo. To determine the downstream molecular mechanism of the circSAMD4A/miR-29c-3p axis, the AMPK/mTOR signaling cascade, which has been reported to be engaged in autophagy (23), was investigated. Western 

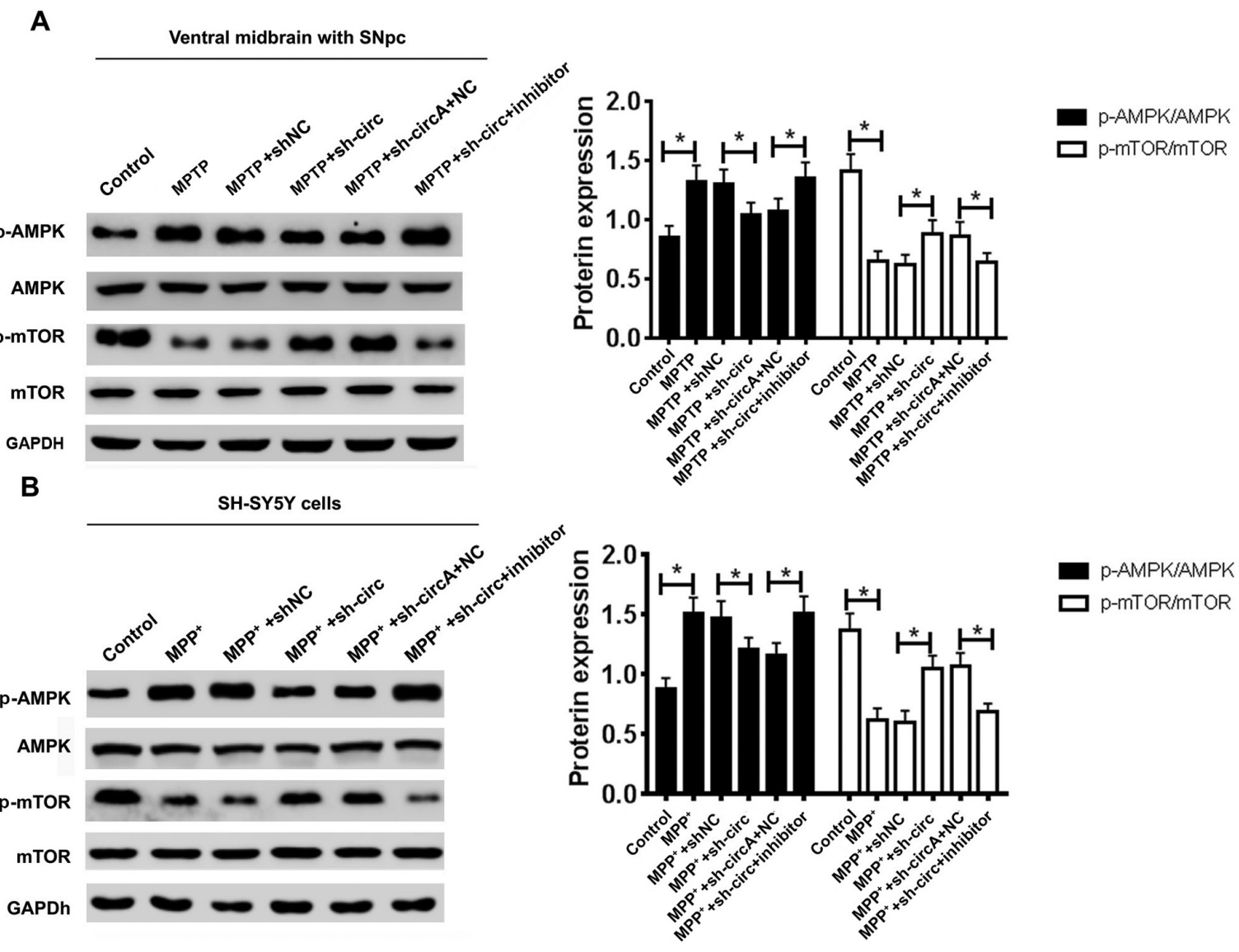

Figure 6. A miR-29c-3p inhibitor abrogates the regulatory effects of sh-circ on the AMPK/mTOR pathway. Effects of circSAMD4A knockdown and miR-29c-3p inhibitor on the protein expression levels of p-AMPK, AMPK, p-mTOR and mTOR were determined via western blotting in both a (A) PD animal model and (B) PD cellular model. "P<0.05. sh-circ, sh-circSAMD4A; miR, microRNA; circSAMD4A, circular RNA sterile $\alpha$ motif domain containing 4A; sh, short hairpin RNA; NC, negative control; MPP', 1-methyl-4-phenylpyridinium; MPTP, 1-methyl-4-phenyl-1,2,3,6-tetrahydropyridine; SNpc, substantia nigra pars compacta; p-, phosphorylated; AMPK, 5'AMP-activated protein kinase; PD, Parkinson's' disease.

blotting results demonstrated that MPTP treatment resulted in increased expression level of p-AMPK and decreased expression level of $\mathrm{p}-\mathrm{mTOR}$ in the midbrain tissues of mice (Fig. 6A). Knockdown of circSAMD4A reversed the upregulation of p-AMPK and downregulation of p-mTOR induced by MPTP in vivo, and the miR-29c-3p inhibitor abolished the effect of circSAMD4A knockdown (Fig. 6A). Consistently, $\mathrm{MPP}^{+}$treatment significantly increased the expression level of $\mathrm{p}$-AMPK and decreased the expression level of p-mTOR in SH-SY5Y cells (Fig. 6B). Knockdown of circSAMD4A reversed the upregulation of $\mathrm{p}$-AMPK and downregulation of $\mathrm{p}-\mathrm{mTOR}$ induced by $\mathrm{MPP}^{+}$in vitro, whereas the miR-29c-3p inhibitor abolished this effect (Fig. 6B). Overall, these results indicated that the miR-29c-3p inhibitor abrogated the regulatory effects of sh-circ on the AMPK/mTOR pathway in vitro and in vivo.

\section{Discussion}

As the mostcommon type of chronic neurodegenerativedisorder, PD currently affects $\sim 3 \%$ of the global population that are $>60$ years of age $(24,25)$. Despite decades of extensive studies, the pathogenesis of PD remains largely unknown. Reduced cell viability, increased cell apoptosis and deficient autophagy flux of neurocytes have been considered to play critical roles in
PD pathogenesis (26-28). To further investigate the underlying pathogenesis of PD, the present study first constructed PD mouse and cellular models. By comparing the application range of C57BL6J and C57BL6 mice, it was discovered that C57BL6 mice are mainly used in tumor, immune, genetic and physiological research, while C57BL6J mice have been used in cardiovascular and cerebrovascular diseases, nervous system diseases, metabolic and developmental diseases, and immune and genetic diseases (29). PD is a nervous system disease. Moreover, the present study consulted the relevant data on the preparation of PD mouse models, and found that C57BL6J mice were one of the main mouse varieties used for PD model establishment (30-35). Therefore, in the current study, the PD mouse model was constructed using C57BL/6J mice. Furthermore, the PD cell model was created using SH-SY5Y cells, based on previous studies $(36,37)$.

Previous studies have shown that circRNAs can regulate synaptic gene expression, cognition ability and memory storage $(38,39)$. Recently, circ-DLG associated protein 4 has been reported to exhibit neuroprotective effects on PD by modulating cAMP responsive element binding protein 1 via miR-134-5p (40). Moreover, in a transgenic C.elegans PD model, Kumar et al (41) revealed that knockdown of circular ZIP-2 decreased the aggregation of $\alpha$-synuclein protein and increased the lifespan by regulating the Daf-16 cascade via 
miR-60. In the present study, it was found that circSAMD4A expression was increased in the MPTP-induced PD animal model and the $\mathrm{MPP}^{+}$-induced SH-SY5Y cell model. In vitro and in vivo experiments also revealed that knockdown of circSAMD4A repressed apoptosis and autophagy, and thereby attenuated the cellular toxicity of MPTP or $\mathrm{MPP}^{+}$toward dopamine neurons or SH-SY5Y cells. Overall, these findings suggested that circSAMD4A may be a useful diagnostic biomarker and therapeutic target for PD.

Previous studies have reported that circRNAs can serve as miRNA sponges to regulate the expression of specific genes (42). In addition, miRNAs have been shown to play a role during PD pathogenesis (43). For example, miR-7 can directly inhibit the expression of $\alpha$-synuclein, whose aggregation is a specific PD marker (44). ciRS-7, one of the most well-studied circRNAs in the central nervous system, has been found to be closely associated with the pathogenesis of AD (45). Recently, miR-7 transfection was used to induce more efficient $\alpha$-synuclein inhibition in a HeLa cell line without ciRS-7 expression, suggesting that ciRS-7 may regulate $\alpha$-synuclein expression in a miR-7-dependent manner, which correlated with the pathogenesis of PD (46). Additionally, circSNCA RNA has been shown to suppress autophagy and promote apoptosis in SH-SY5Y cells by facilitating the expression of $\alpha$-synuclein mRNA via sponging miR-7 (47). In the present study, miR-29c-3p was found to be targeted and regulated by circSAMD4A. Although miR-29c-3p has been reported to participate in the pathogenesis of multiple human diseases, such as colorectal cancer, ovarian cancer and AD (48-50), to the best of our knowledge, its role in PD has not been previously reported. In the current study, miR-29c-3p was identified to abolish the protective effects of circSAMD4A knockdown against MPTP- or $\mathrm{MPP}^{+}$-induced apoptosis and autophagy.

AMPK is a key cell receptor that is responsible for monitoring alterations in energy regulation, while mTOR is a vital signaling complex involved in numerous cellular processes (23). Both AMPK and mTOR have been reported to be associated with the regulation of apoptosis and autophagy during tumor progression $(51,52)$. Moreover, miR-124 was found to protect dopaminergic neurons by modulating apoptosis and autophagy via the regulation of the AMPK/mTOR cascade in PD (53). Subsequently, the long non-coding RNA metastasis associated lung adenocarcinoma transcript 1 was revealed to contribute to cellular apoptosis in PD by targeting miR-124 (19). The present study demonstrated that knockdown of circSAMD4A significantly reversed the upregulation of $\mathrm{p}$-AMPK and downregulation of $\mathrm{p}-\mathrm{mTOR}$ in an MPTP-induced animal PD model and $\mathrm{MPP}^{+}$-induced PD cell PD, which was abolished by transfection with a miR-29c-3p inhibitor.

There were some limitations to the present study. First, the positive results assessing the function of circRNAs in PD were limited, Second, there was a lack of clinical studies to verify the results. Therefore, further studies will be needed.

In conclusion, the present study indicated that circSAMD4A participated in apoptosis and autophagy of dopaminergic neurons by regulating the AMPK/mTOR pathway via miR-29c-3p in PD. The current findings may nonetheless contribute to the development of effective candidate drugs for PD treatment.

\section{Acknowledgements}

Not applicable.

\section{Funding}

No funding was received.

\section{Availability of data and materials}

The datasets used and/or analyzed during the current study are available from the corresponding author on reasonable request.

\section{Authors' contributions}

WSW participated in the study design and manuscript writing. RXL performed experiments, analyzed data and participated in manuscript writing. JJZ participated in statistical analysis and manuscript writing. YL performed experiments and analyzed data. WSW and YL confirm the authenticity of all the raw data. All authors read and approved the final manuscript.

\section{Ethics approval and consent to participate}

All animal experiments followed stated protocols from the Research Committee of Ningbo No. 6 Hospital and permission was obtained from the Animal Experiment Center of the Institute of Radiation Medicine of the Chinese Academy of Medical Sciences [permit no. SCXK(JING)2019-0010].

\section{Patient consent for publication}

Not applicable.

\section{Competing interests}

The authors declare that they have no competing interests.

\section{References}

1. Beitz JM: Parkinson's disease: A review. Front Biosci (Schol Ed) 6: 65-74, 2014.

2. Tysnes OB and Storstein A: Epidemiology of Parkinson's disease. J Neural Transm (Vienna) 124: 901-905, 2017.

3. Schrag A, Hovris A, Morley D, Quinn N and Jahanshahi M: Young-vs. older-onset Parkinson's disease: Impact of disease and psychosocial consequences. Mov Disord 18: 1250-1256, 2003.

4. Stern M, Dulaney E, Gruber SB, Golbe L, Bergen M, Hurtig H, Gollomp S and Stolley P: The epidemiology of Parkinson's disease: A case-control study of young-onset and old-onset patients. Arch Neurol 48: 903-907, 1991.

5. Elbaz A, Carcaillon L, Kab S and Moisan F: Epidemiology of Parkinson's disease. Rev Neurol (Paris) 172: 14-26, 2016.

6. Schneider RB, Iourinets J and Richard IH: Parkinson's disease psychosis: Presentation, diagnosis and management. Neurodegener Dis Manag 7: 365-376, 2017.

7. Orimo S: New development of diagnosis and treatment for Parkinson's disease. Rinsho Shinkeigaku 57: 259-273, 2017 (In Japanese).

8. Salzman J: Circular RNA Expression: Its potential regulation and function. Trends Genet 32: 309-316, 2016.

9. Hsiao KY, Sun HS and Tsai SJ: Circular RNA-New member of noncoding RNA with novel functions. Exp Biol Med (Maywood) 242: 1136-1341, 2017. 
10. Jahani S, Nazeri E, Majidzadeh AK, Jahani M and Esmaeili R: Circular RNA; a new biomarker for breast cancer: A systematic review. J Cell Physiol 235: 5501-5510, 2020.

11. Zhang HD, Jiang LH, Sun DW, Hou JC and Ji ZL: CircRNA: A novel type of biomarker for cancer. Breast Cancer 25: 1-7, 2018

12. Zhao ZJ and Shen J: Circular RNA participates in the carcinogenesis and the malignant behavior of cancer. RNA Biol 14: 514-521, 2017.

13. Wu F, Han B, Wu S, Yang L, Leng S, Li M, Liao J, Wang G, Ye Q, Zhang Y, et al: Circular RNA TLK1 aggravates neuronal injury and neurological deficits after ischemic stroke via miR-335-3p/TIPARP. J Neurosci 39: 7369-7393, 2019.

14. Bai Y, Zhang Y, Han B, Yang L, Chen X, Huang R, Wu F, Chao J, Liu P, Hu G, et al: Circular RNA DLGAP4 ameliorates ischemic stroke outcomes by targeting mir-143 to regulate endothelial-mesenchymal transition associated with blood-brain barrier integrity. J Neurosci 38: 32-50, 2018.

15. Gong GH, An FM, Wang Y, Bian M, Wang D and Wei CX: Comprehensive Circular RNA profiling reveals the regulatory role of the CircRNA-0067835/miR-155 pathway in temporal lobe epilepsy. Cell Physiol Biochem 51: 1399-1409, 2018.

16. Akhter R: Circular RNA and Alzheimer's disease. Adv Exp Med Biol 1087: 239-243, 2018

17. Yang H, Wang H, Shang H, Chen X, Yang S, Qu Y, Ding J and Li X: Circular RNA circ_0000950 promotes neuron apoptosis, suppresses neurite outgrowth and elevates inflammatory cytokines levels via directly sponging miR-103 in Alzheimer's disease. Cell Cycle 18: 2197-2214, 2019.

18. Zhao Y and Jing Z: CircSAMD4A accelerates cell proliferation of osteosarcoma by sponging miR-1244 and regulating MDM2 mRNA expression. Biochem Biophys Res Commun 516: 102-111, 2019.

19. Liu W, Zhang Q, Zhang J, Pan W, Zhao J and Xu Y: Long non-coding RNA MALAT1 contributes to cell apoptosis by sponging miR-124 in Parkinson disease. Cell Biosci 7: 19, 2017.

20. Paschall AV and Liu K: An orthotopic mouse model of spontaneous breast cancer metastasis. J Vis Exp 54040, 2016.

21. Creamer-Hente MA, Lao FK, Dragos ZP and Waterman LL: Sex- and Strain-related differences in the stress response of Mice to $\mathrm{CO}_{2}$ euthanasia. J Am Assoc Lab Anim Sci 57: 513-519, 2018

22. Livak KJ and Schmittgen TD: Analysis of relative gene expression data using real-time quantitative PCR and the 2(-Delta Delta $\mathrm{C}(\mathrm{T})$ ) method. Methods 25: 402-408, 2001

23. Kim J, Kundu M, Viollet B and Guan KL: AMPK and mTOR regulate autophagy through direct phosphorylation of Ulk1. Nat Cell Biol 13: 132-141, 2011.

24. Hirsch L, Jette N, Frolkis A, Steeves T and Pringsheim T: The incidence of Parkinson's disease: A systematic review and meta-analysis. Neuroepidemiology 46: 292-300, 2016.

25. Shalash AS, Hamid E, Elrassas HH, Bedair AS, Abushouk AI, Khamis M, Hashim M, Ahmed NS, Ashour S and Elbalkimy M: Non-motor symptoms as predictors of quality of life in egyptian patients with parkinson's disease: A cross-sectional study using a culturally adapted 39-item parkinson's disease questionnaire. Front Neurol 9: 357, 2018

26. Cui B, Guo X, You Y and Fu R: Farrerol attenuates MPP+ -induced inflammatory response by TLR 4 signaling in a microglia cell line. Phytother Res 33: 1134-1141, 2019.

27. Malley KR, Koroleva O, Miller I, Sanishvili R, Jenkins CM, Gross RW and Korolev S: The structure of iPLA $\beta$ reveals dimeric active sites and suggests mechanisms of regulation and localization. Nat Commun 9: 765, 2018.

28. Suresh SN, Chavalmane AK, Dj V, Yarreiphang H, Rai S, Paul A, Clement JP, Alladi PA and Manjithaya R: A novel autophagy modulator 6-Bio ameliorates SNCA/ $\alpha$-synuclein toxicity. Autophagy 13: 1221-1234, 2017.

29. Sanduzzi Zamparelli M, Compare D, Coccoli P, Rocco A, Nardone OM, Marrone G, Gasbarrini A, Grieco A, Nardone G and Miele L: The metabolic role of gut microbiota in the development of nonalcoholic fatty liver disease and cardiovascular disease. Int J Mol Sci 17: 1225, 2016.

30. Ramalingam M, Huh YJ and Lee YI: The impairments of $\alpha$-synuclein and mechanistic target of rapamycin in rotenone-induced SH-SY5Y cells and mice model of Parkinson's disease. Front Neurosci 13: 1028, 2019.

31. Pupyshev AB, Tikhonova MA, Akopyan AA, Tenditnik MV, Dubrovina NI and Korolenko TA: Therapeutic activation of autophagy by combined treatment with rapamycin and trehalose in a mouse MPTP-induced model of Parkinson's disease. Pharmacol Biochem Behav 177: 1-11, 2019.
32. Ge G, Chen C, Guderyon MJ, Liu J, He Z, Yu Y, Clark RA and $\mathrm{Li}$ S: Regulatable lentiviral hematopoietic stem cell gene therapy in a mouse model of Parkinson's disease. Stem Cells Dev 27: 995-1005, 2018.

33. de Campos PS, Kawamura L, Hasegawa K, Kumei Y and Zeredo JL: Analysis of respiratory movements in a mouse model of late Parkinson's disease submitted to stress. Respir Physiol Neurobiol 251: 50-56, 2018.

34. Sugumar M, Sevanan M and Sekar S: Neuroprotective effect of naringenin against MPTP-induced oxidative stress. Int J Neurosci 129: 534-539, 2019.

35. Zhang X, Zhang Y, Li R, Zhu L, Fu B and Yan T: Salidroside ameliorates Parkinson's disease by inhibiting NLRP3-dependent pyroptosis. Aging (Albany NY) 12: 9405-9426, 2020.

36. Sang Q, Liu X, Wang L, Qi L, Sun W, Wang W, Wang W, Sun Y and Zhang H: Curcumin protects an SH-SY5Y cell model of parkinson's disease against toxic injury by regulating HSP90. Cell Physiol Biochem 51: 681-691, 2018.

37. Xicoy H, Wieringa B and Martens GJ: The SH-SY5Y cell line in Parkinson's disease research: A systematic review. Mol Neurodegener 12: 10, 2017.

38. Zimmerman AJ, Hafez AK, Amoah SK, Rodriguez BA, Dell'Orco M, Lozano E, Hartley BJ, Alural B, Lalonde J, Chander $\mathrm{P}$, et al: A psychiatric disease-related circular RNA controls synaptic gene expression and cognition. Mol Psychiatry 25: 2712-2727, 2020.

39. Ayers D and Scerri C: Non-coding RNA influences in dementia. Noncoding RNA Res 3: 188-194, 2018.

40. Feng Z, Zhang L, Wang S and Hong Q: Circular RNA circDLGAP4 exerts neuroprotective effects via modulating miR-134-5p/CREB pathway in Parkinson's disease. Biochem Biophys Res Commun 522: 388-394, 2020.

41. Kumar L, Shamsuzzama, Jadiya P, Haque R, Shukla S and Nazir A: Functional characterization of novel circular RNA molecule, circzip-2 and its synthesizing gene zip-2 in C. Elegans model of parkinson's disease. Mol Neurobiol 55: 6914-6926, 2018.

42. Xiao MS, Ai Y and Wilusz JE: Biogenesis and functions of circular RNAs come into focus. Trends Cell Biol 30: 226-240, 2020.

43. Leggio L, Vivarelli S, L'Episcopo F, Tirolo C, Caniglia S, Testa N, Marchetti B and Iraci N: microRNAs in Parkinson's disease: From pathogenesis to novel diagnostic and therapeutic approaches. Int J Mol Sci 18: 2698, 2017.

44. Junn E, Lee KW, Jeong BS, Chan TW, Im JY and Mouradian MM: Repression of alpha-synuclein expression and toxicity by microRNA-7. Proc Natl Acad Sci USA 106: 13052-13057, 2009.

45. Lukiw WJ: Circular RNA (circRNA) in Alzheimer's disease (AD). Front Genet 4: 307, 2013.

46. Hansen TB, Jensen TI, Clausen BH, Bramsen JB, Finsen B, Damgaard CK and Kjems J: Natural RNA circles function as efficient microRNA sponges. Nature 495: 384-388, 2013.

47. Sang Q, Liu X, Wang L, Qi L, Sun W, Wang W, Sun Y and Zhang H: CircSNCA downregulation by pramipexole treatment mediates cell apoptosis and autophagy in Parkinson's disease by targeting miR-7. Aging (Albany NY) 10: 1281-1293, 2018.

48. Wu Y, Xu J, Xu J, Cheng J, Jiao D, Zhou C, Dai Y and Chen Q: Lower serum levels of miR-29c-3p and miR-19b-3p as biomarkers for alzheimer's disease. Tohoku J Exp Med 242: 129-136, 2017.

49. Zhang S, Jin J, Tian X and Wu L: hsa-miR-29c-3p regulates biological function of colorectal cancer by targeting SPARC. Oncotarget 8: 104508-104524, 2017

50. Luo H, Zhu W, Mo W and Liang M: High-glucose concentration aggravates TNF-alpha-induced cell viability reduction in human CD146-positive periodontal ligament cells via TNFR-1 gene demethylation. Cell Biol Int 44: 2383-2394, 2020.

51. Takagi H, Matsui Y, Hirotani S, Sakoda H, Asano $T$ and Sadoshima J: AMPK mediates autophagy during myocardial ischemia in vivo. Autophagy 3: 405-407, 2007.

52. Lu C, Wang W, Jia Y, Liu X, Tong Z and Li B: Inhibition of AMPK/autophagy potentiates parthenolide-induced apoptosis in human breast cancer cells. J Cell Biochem 115: 1458-1466, 2014

53. Gong X, Wang H, Ye Y, Shu Y, Deng Y, He X, Lu G and Zhang S: miR-124 regulates cell apoptosis and autophagy in dopaminergic neurons and protects them by regulating AMPK/mTOR pathway in Parkinson's disease. Am J Transl Res 8: 2127-2137, 2016.

This work is licensed under a Creative Commons

Attribution-NonCommercial-NoDerivatives 4.0 International (CC BY-NC-ND 4.0) License. 